\title{
¿Existe una relación entre los programas de mejora de la calidad y la satisfacción de usuarias de atención prenatal?: experiencia en hospitales del Perú
}

\author{
Juan A. Seclen-Palacín, ${ }^{1}$ Bruno Benavides, ${ }^{2}$ Enrique Jacoby, ${ }^{3}$ \\ Aníbal Velásquez ${ }^{4}$ y Enrique Watanabe ${ }^{5}$
}

Forma de citar

Seclen-Palacín JA, Benavides B, Jacoby E, Velásquez A, Watanabe E. ¿Existe una relación entre los programas de mejora de la calidad y la satisfacción de usuarias de atención prenatal?: experiencia en hospitales del Perú. Rev Panam Salud Publica. 2004;16(3):149-57.

RESUMEN Objetivos. 1) Determinar el nivel de satisfacción de las usuarias (SU) de control prenatal en hospitales donde se implementó un programa de mejora continua de la calidad (PMC), y comparar la SU en estos pacientes y en un grupo de referencia. 2) Estudiar comparativamente las razones de insatisfacción en ambos grupos. 3) Identificar los factores asociados a la SU y estudiar su relación con el programa de calidad.

Métodos. Estudio cuasiexperimental con una muestra representativa de gestantes que acudieron a atención prenatal hospitalaria. Se aplicaron encuestas de salida en el grupo de intervención $(\mathrm{n}=191)$ y el grupo de referencia $(\mathrm{n}=185)$. La variable dependiente fue la satisfacción con la atención prenatal, y las independientes fueron satisfacción de expectativas, trato del personal, nivel de información, equipamiento, percepción del tiempo de espera, limpieza y comodidad. Se realizaron análisis estadísticos descriptivos y multivariados para responder a los objetivos.

Resultados. En el grupo de pacientes que acudieron a los hospitales que participaron en el PMC se encontraron mayores niveles de SU $(67,5 \%$ frente al $55,1 \%$ en el grupo de referencia, $\mathrm{P}=0,014)$. Las razones de insatisfacción fueron el tiempo de espera prolongado y el trato inadecuado, siendo estas últimas más frecuentes en el grupo de referencia. El análisis multivariado indicó que el trato del personal, la información durante la consulta, la satisfacción de las expectativas y la aplicación del PMC fueron factores que mostraron una asociación estadísticamente significativa con la SU.

Conclusiones. Se encontró que el PMC en los servicios maternos y perinatales tuvo una asociación positiva con la SU. Asimismo, los resultados refuerzan la necesidad de ofertar servicios humanizados de atención prenatal basados en el buen trato y en la información al usuario, con la idea de mejorar la aceptabilidad de los servicios de salud y la adherencia de la gestante a ellos.

Palabras clave Satisfacción de usuario, calidad de atención prenatal, evaluación de servicios.

1 Consultor en sistemas y servicios de salud, Organización Panamericana de la Salud, Representación en el Brasil (hasta junio de 2004). Consultor externo, Organización Panamericana de la Salud, Representación en el Perú (a partir de julio de 2004). La correspondencia debe dirigirse a: Los Cedros 269, San Isidro Lima 27, Perú, Casilla Postal 2117, Lima 100, Perú; Correo electrónico: jseclen@yahoo. com
2 Oficial del Programa para la Región de América Latina, Partners in Population and Development, Dhaka, Bangladesh.

3 Médico Asesor Regional de la Unidad de Nutrición, Organización Panamericana de la Salud, Washington, D.C., Estados Unidos de América.
4 Consultor en evaluación, Profesor del Departamento de Medicina Preventiva, Universidad Nacional Mayor de San Marcos, Lima, Perú.

Sociólogo, consultor en salud pública, Lima, Perú. 
La satisfacción del usuario (SU) es un tema de interés creciente en el marco de la salud pública y de la gestión de los servicios de salud (1); se considera un eje angular de la evaluación de servicios de salud (2) y un resultado de la calidad de la asistencia $(3,4)$ y de su efectividad (5). Su conceptualización y entendimiento causal son complejos y múltiples porque residen en la percepción subjetiva del usuario, y su valoración se basa en métodos, técnicas e instrumentos de medición diversos (6-9) y poco estandarizados (10). Su estudio es de utilidad ya que se ha encontrado una relación entre la satisfacción con la utilización de los servicios (11), la conducta terapéutica del paciente y la continuidad de la atención (12). La SU depende de factores individuales (13), de la calidad del servicio y tecnología disponible (14), del entorno familiar y del contexto social en el que se desarrolla la interacción entre el usuario y el servicio de salud $(15,16)$.

Existen varias experiencias en torno a la calidad en los hospitales, y redes de atención primaria de salud que consideran como uno de sus ejes de intervención la SU, entendida esta como una medida de aceptabilidad social $(1,13,14)$. En América Latina, durante las últimas décadas se han desarrollado varias iniciativas y programas de calidad (17) que, aplicados a los servicios de salud, han contribuido a mejorar la capacidad resolutiva de los servicios y la satisfacción de las necesidades sanitarias de la población.

En el Perú, desde los inicios de los años noventa se han desarrollado algunas intervenciones de mejora de la calidad en establecimientos y servicios de salud, con el fin de afrontar graves problemas sanitarios como la todavía elevada tasa de mortalidad infantil, la mortalidad materna y el limitado acceso a servicios resolutivos, confiables y humanizados (18). El Proyecto 2000 constituye una de las iniciativas para mejorar la salud materna y neonatal en zonas criticas del país (19).

El presente informe comunica algunos de los resultados obtenidos tras la evaluación de la primera etapa del Proyecto 2000 (20). Se presentan los resultados de una de las dimensiones de la evaluación: la aceptabilidad. Los objetivos de este trabajo fueron 1) determinar el nivel de SU con el control prenatal de los hospitales donde se implementó un programa de mejora continua de la calidad (PMC) y compararla con la SU en un grupo de referencia, 2) estudiar comparativamente las razones de insatisfacción en ambos grupos de estudio y, 3) identificar los factores asociados a la SU y estudiar su relación con el PMC.

\section{MÉTODOS}

\section{Ámbito de estudio}

Perú, país ubicado en Sudamérica, tiene una población de 26 millones de habitantes, de los cuales el $65 \%$ viven en espacios urbanos. El cincuenta y cuatro por ciento de la población total es pobre, y la tasa de analfabetismo en mayores de 15 años es del 7\%. Lima, la capital, está situada en el litoral occidental del Océano Pacífico y tiene 7,4 millones de habitantes (21). Los datos de la situación de salud reproductora muestran que la razón de mortalidad materna es de $265 \times 100000$ nacidos vivos, la cobertura de partos atendidos por personal de salud es del $56,4 \%$, el control prenatal alcanza el $67,2 \%$ de la población, y la prevalencia del uso de anticonceptivos modernos en mujeres con pareja fue del 41,3\% en 1996 (22).

\section{La intervención}

Se desarrolló un programa de PMC en servicios maternos y perinatales, denominado Proyecto 2000, orientado a mejorar la salud materna y del neonato y a contribuir a la reducción de la mortalidad materna. El programa tenía como propósito mejorar la calidad de los procesos de atención prenatal, parto, control del puerperio y manejo de las complicaciones maternas y neonatales. El PMC se aplicó, en su primera etapa, en 89 hospitales y centros de salud de mediana complejidad (de 1996 al primer semestre del 2000); y en su etapa de consolidación (segundo semestre del 2000 a 2002) en 30 estableci- mientos pertenecientes a las 12 Direcciones de Salud (DISA) intervenidas.

La intervención estuvo orientada a mejorar la calidad en tres aspectos: accesibilidad, efectividad y aceptabilidad de los servicios obstétricos y perinatales (23). La SU fue uno de los indicadores de la dimensión de aceptabilidad (figura 1). El énfasis del PMC se centró en la organización de los servicios en cinco procesos de organización y prestación:

1) el uso de datos, relacionado con el proceso de recolección y procesamiento de datos y el uso de la información para la toma de decisiones gerenciales y clínicas del área materna y perinatal;

2) el manejo clínico estandarizado, el cual promovía la estandarización y protocolización de los procedimientos médicos de atención obstétrica y neonatal;

3) la educación permanente en servicio, basado en el análisis de grupos y horizontal que el personal de salud realizaba sobre la práctica diaria de los procesos de atención materna y perinatal, de modo que pudieran identificar oportunidades de intervención para mejorar sus acciones, prácticas y procedimientos de trabajo;

4) la gestión y disponibilidad de recursos, orientado hacia la optimización de la gestión local para el suministro adecuado y oportuno de insumos, equipos y medicamentos básicos para la atención; y

5) la relación entre el proveedor, el usuario y la comunidad, destinada a favorecer el acceso precoz, oportuno y continuo de la gestante hacia los servicios obstétricos mediante estrategias de comunicación eficaces entre personas y de educación de salud, la mejora de la cordialidad, el trato, el respeto y la empatía hacia las usuarias. Al mejorar esta relación, se facilita el desarrollo conjunto, de parte de los servicios, los agentes locales de salud y la comunidad, de acciones en pro de la salud de la mujer embarazada.

Los procesos de trabajo estimulados por el PMC fueron las reuniones mul- 
FIGURA 1. Modelo de la intervención empleada en el Programa de Mejora Continua (PMC). Evaluación de un programa de calidad en hospitales del Perú (2000). Versión adaptada de lo expuesto en la evaluación final del programa (19)

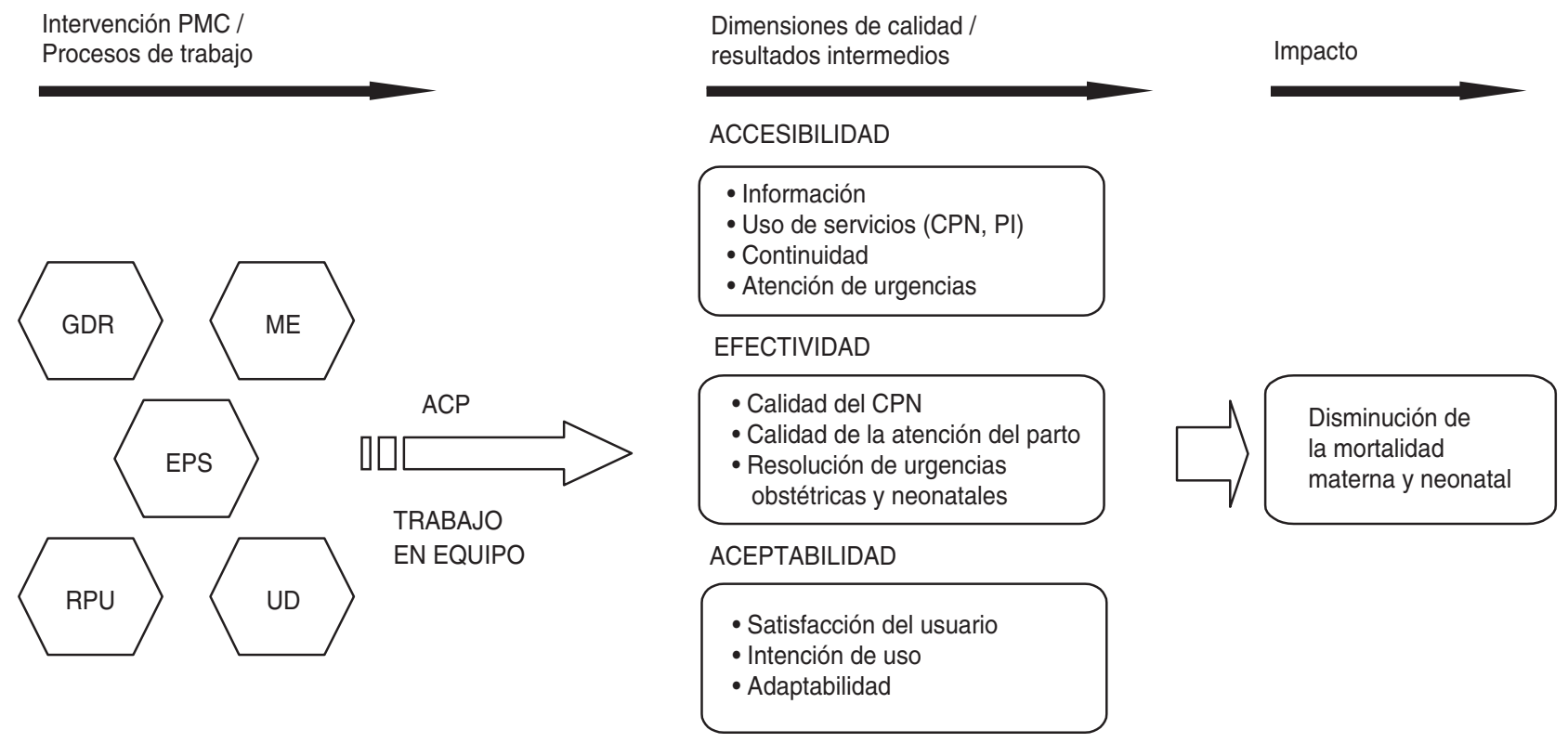

GDR = Gestión y disponibilidad de recursos; ME = Manejo estandarizado; EPS = Educación permanente en salud; RPU = Relación proveedor usuario comunidad; UD = Uso de datos; $\mathrm{ACP}=$ Análisis crítico de la práctica; $\mathrm{CPN}=$ Control prenatal; $\mathrm{PI}=$ Parto institucional.

tidisciplinarias y de trabajo en equipo, el análisis crítico de la práctica diaria (problematización), la adopción de decisiones gerenciales basada en información, las sesiones de autosupervisión, el seguimiento de usuarias, la incorporación de percepciones culturales de la población en la oferta de servicios, las estrategias de comunicación entre personas durante la consulta obstétrica, el trabajo comunitario, la humanización de la atención, y la estandarización y revisión continua de los procedimientos clínicos y quirúrgicos obstétricos y neonatales.

\section{Diseño del estudio}

Usamos un diseño cuasiexperimental para conformar dos grupos de estudio. El grupo de intervención (PMC) se compuso de 18 hospitales donde se implementó el programa, y el grupo de referencia estuvo compuesto de 19 hospitales que no participaron en el PMC. La evaluación se realizó al final de la primera etapa del Proyecto (primer semestre 2000). Las encuestas se aplica- ban cuando las gestantes salían de la consulta prenatal.

\section{Población de estudio}

La población de estudio estuvo compuesta de gestantes que egresaron de la consulta prenatal del servicio de obstetricia de hospitales públicos del Perú. Participaron 191 mujeres en el grupo de hospitales PMC, y 185 en el grupo sin PMC.

\section{Selección de la muestra}

Siendo el propósito de la selección la conformación de grupos comparables, se utilizó un método de muestreo multietápico, estratificado, aleatorizado y proporcional. En primer lugar, para cada una de las DISA se eligieron variables de la Encuesta Nacional de Demografía y Salud 2000 (ENDES 2000) y el Mapa de Pobreza 2000 elaborado por una institución pública peruana denominada Fondo de Compensación y Desarrollo Social (FONCODES). Dichas variables fueron: proporción de mujeres en edad fértil analfabetas, prevalencia del uso de métodos de anticoncepción, cobertura de partos institucionales, prevalencia de desnutrición infantil, y niveles de pobreza. Cada una de estas variables tuvo una escala de puntuación numérica que sirvió de referencia, a través de una sumatoria simple, para la definición de tres estratos (alto, medio y bajo) para las 34 DISA que tiene el sistema de salud peruano. Luego se seleccionó por cada DISA con PMC, una DISA de referencia en función de los estratos establecidos. Acto seguido, considerando el número de hospitales de cada DISA con PMC (1 ó 2 según el caso) se seleccionó su respectivo hospital de referencia. De esta forma, quedaron conformados dos grupos de estudio: el de hospitales con PMC y sin PMC, según DISA y estrato. En la DISA Piura, perteneciente al grupo sin PMC, solo se disponía de un hospital en tanto que en su DISA correspondiente con PMC existían dos hospitales; por tal motivo en este último grupo existe un hospital más que en el grupo de referencia (sin PMC). 
Después de este proceso, se procedió a determinar el número de entrevistas que deberían realizarse por cada grupo de estudio. En tal sentido, considerando el grupo de hospitales como la unidad de muestreo, el 0,5 como nivel de satisfacción de las gestantes, un error del $5 \%$, y el $80 \%$ como potencia del estudio, se estimó que el número de gestantes a entrevistar en cada grupo debería ser de 170 (24). Con la finalidad de evitar posibles pérdidas de muestra durante el trabajo de campo se consideró necesario adicionar un $10 \%$ de entrevistas; por ello el tamaño final de la muestra fue de 185 gestantes por cada grupo como mínimo. Finalmente, las gestantes fueron escogidas en cada uno de los grupos según un muestreo por saturación, considerando un total de 10 gestantes a entrevistar por cada hospital de estudio.

\section{Instrumento}

Se utilizó un cuestionario validado. En su diseño participaron funcionarios y técnicos del Ministerio de Salud, consultores del PMC y personal de los servicios de atención maternoperinatal de hospitales. La validación se realizó en la ciudad de Lima. La encuesta incluyó secciones correspondientes a datos personales, socioeconómicos, educacionales y de vivienda, y también las expectativas de las usuarias hacia la atención prenatal. La pregunta relacionada con la satisfacción fue: "Señora, ¿qué le pareció la atención que le dieron en el hospital? ¿Por qué?". Luego se exploró la satisfacción sobre cada uno de los procesos de la atención (trato, información recibida, tiempo de espera, equipos, limpieza, local ente otros). Antes de terminar la entrevista el encuestador empleó una lista de chequeo para indagar si la atención recibida fue satisfactoria para cada una de las expectativas que la usuaria había expresado al inicio de la entrevista: "Señora, ¿le dieron [. . . ] conforme usted lo esperaba?". Las expectativas colocadas en el cuestionario fueron elegidas por decisión de un grupo focal. Se incluyeron las expectativas sobre el trato del personal de salud, la realización de algunos procedimientos (determinación del peso y talla, toma de presión arterial, análisis de laboratorio, ecografías, etc.), curso del embarazo, crecimiento, posición y salud del bebé, acompañamiento de familiares durante la atención prenatal, escucha de latidos del bebé, examen minucioso, la no realización de tacto vaginal durante la consulta, atención por prestador femenino, orientación sobre la alimentación, prescripción de vitaminas, acceso a los resultados de los exámenes de laboratorio previos, el no pago por los servicios, precios baratos, limpieza del personal que atiende y del local de la atención, y buen equipamiento. Aquellas expectativas no incluidas en este listado también fueron registradas.

\section{Mediciones}

La variable dependiente fue la satisfacción de la usuaria con la atención prenatal, definida esta como la percepción de la entrevistada sobre la atención que recibió. La satisfacción se consideró aceptable cuando las respuestas eran "muy buena" y "buena" según la escala de Likert, mientras que las respuestas "muy mala", "mala" y "regular" fueron consideradas como insatisfacción (variable dependiente binaria). Las variables de ajuste fueron demográficas (edad), socioeconómicas (condición del techo, paredes y piso de la vivienda de la usuaria, tenencia de artefactos eléctricos, acceso a servicios de saneamiento); educacional (analfabeta, primaria, secundaria o superior); aseguramiento de salud (seguro público de atención maternoinfantil, seguridad social, seguro privado); y continuidad en la atención con los servicios públicos de salud (consulta de primera vez y consultas posteriores). Las variables independientes, es decir aquellas que se esperaba tuvieran alguna relación con la SU, fueron las percepciones sobre la información proporcionada en el control prenatal, el trato, la confortabilidad del local, los equipos médicos, la limpieza del establecimiento y el tiempo de espera para la atención percibido. Todas estas variables fueron medidas en escala ordinal de 5 categorías (desde el nivel muy bueno hasta el muy malo). Se consideró también la variable satisfacción de expectativas, la cual estuvo definida como la relación entre las expectativas de las entrevistadas antes de llegar a la consulta y el grado de satisfacción de las mismas con la atención. La medición se hizo en escala numérica, y su valor fue de 0 a 1 , calculado mediante una división entre el número de expectativas satisfechas por la atención prenatal y el número total de expectativas previas a la llegada al hospital expresadas espontáneamente por la usuaria. La variable grupo de estudio fue considerada binaria $(1=$ grupo de intervención con PMC y $0=$ grupo de referencia sin PMC).

\section{Análisis estadístico}

Se utilizó un análisis descriptivo de frecuencias simples que comparaban la distribución y magnitud de las variables en los dos grupos de estudio. Para estimar las diferencias entre grupos se utilizó la prueba U de Mann-Whitney (para variables de distribución no paramétrica y ordinales) y la prueba del ji cuadrado (para variables categóricas). Para la estimación de los factores asociados a la satisfacción se utilizó el análisis multivariado del tipo regresión logística múltiple, realizándose pruebas de colinearidad (para excluir del modelo los variables cuyo coeficiente de correlación fuese mayor de 0,7) y análisis de residuos. Se consideró como diferencia significativa el valor $P<0,05$. Los datos fueron ingresados con doble entrada en el programa Epi-info 6.04, siendo la base datos almacenada en formato dBASE. El análisis estadístico en sí fue realizado con el programa SPSS versión 10.0.

\section{RESULTADOS}

\section{Características generales}

Se estudiaron 191 gestantes que egresaron de la consulta prenatal en el grupo PMC y 185 en el grupo de referencia. La edad media de las entrevistadas fue 25,4 años $(\mathrm{DE}=6,2)$ en el grupo PMC, y 25,9 años (DE =6,7) 
en el grupo de referencia. No se hallaron diferencias significativas entre los grupos según edad, nivel educativo, alumbrado público, acceso a saneamiento básico, continuidad en la atención, y cobertura por seguro de salud (cuadro 1).

\section{Expectativas de las usuarias}

En el grupo PMC se encontró que el promedio fue 1,49 expectativas por gestante mientras que en el grupo de referencia el promedio fue 1,64; esta diferencia no fue significativa según la prueba U de Mann-Whitney, con un valor de $P=0,083$. También se midió el grado de satisfacción de las expectativas, para lo cual se calculó una razón entre el número de expectativas satisfechas y el número total de expectativas expresada por cada usuaria. Así, en el grupo PMC el promedio de dicho valor fue 0,72 , y en el grupo de referencia fue 0,75 . Esta diferencia no fue significativa según la prueba $U$ de MannWhitney, con un valor de $P=0,573$.

\section{Satisfacción de las usuarias}

En el grupo PMC el 58,6\% de las entrevistadas opinó que la satisfacción con la atención prenatal hospitalaria fue buena, y un 8,9\% opinó que la atención fue muy buena, mientras que en el grupo de referencia dichos niveles fueron del $48,6 \%$ y $6,5 \%$, respectivamente. Las diferencias de proporciones mostraron significación estadística (prueba $\mathrm{U}$ de Mann-Whitney, $P=0,014$ ). En relación con el resto de las variables, se encontró que las usuarias del grupo PMC consideraron que la información recibida durante la consulta prenatal fue buena $(62,3 \%)$ o muy buena $(22,5 \%)$; en el grupo de referencia dichos valores fueron del $64,3 \%$ y $15,1 \%$, respectivamente. Estas diferencias fueron significativas (prueba U de MannWhitney, $P=0,048$ ). En el grupo de las usuarias del control prenatal PMC el trato del personal se consideró bueno $(64,9 \%)$ o muy bueno $(4,2 \%)$, siendo estos valores distintos en el grupo de referencia $(51,4 \%$ y $5,9 \%$ para las mis-

CUADRO 1. Características descriptivas de la población de estudio $(n=376)$. Evaluación de un programa de calidad en hospitales del Perú, 2000

\begin{tabular}{|c|c|c|c|}
\hline Variables & $\begin{array}{c}\text { Grupo PMC } \\
(n=191)\end{array}$ & $\begin{array}{l}\text { Grupo sin PMC } \\
\quad(n=185)\end{array}$ & Valor $P$ \\
\hline Edad (años) & 25,4 (DE 6,2) & 25,9 (DE 6,7) & $0,549^{a}$ \\
\hline \multicolumn{4}{|l|}{ Nivel educacional (\%) } \\
\hline Ninguno & 1,6 & 2,2 & \\
\hline Primaria & 20,5 & 19,5 & \\
\hline Secundaria & 46,3 & 51,9 & \\
\hline Superior & 31,6 & 26,5 & $0,493^{b}$ \\
\hline \multicolumn{4}{|l|}{ Alumbrado eléctrico (\%) } \\
\hline Posee & 88,9 & 91,8 & \\
\hline No posee (tienen otro, queroseno, vela, gas) & 11,1 & 8,2 & $0,352^{a}$ \\
\hline \multicolumn{4}{|l|}{ Saneamiento básico (\%) } \\
\hline Posee red de alcantarillado & 74,4 & 75,7 & \\
\hline No posee red (letrina, pozo ciego, acequia) & 25,6 & 24,3 & $0,302^{\mathrm{a}}$ \\
\hline \multicolumn{4}{|l|}{ Continuidad de la atención (\%) } \\
\hline Primera vez & 23,2 & 28,6 & \\
\hline Consulta posterior & 76,8 & 71,4 & $0,225^{a}$ \\
\hline Disponen de seguro de salud (\%) & 20,4 & 16,8 & $0,362^{a}$ \\
\hline
\end{tabular}

a Valor $P$ estimado con la prueba $U$ de Mann-Whitney.

b Valor $P$ estimado con la prueba de Kruskall Wallis.

mas categorías, $P=0,027$ según la prueba U de Mann-Whitney). En relación al resto de las variables se observó que la opinión de las usuarias sobre la limpieza, los equipos y la confortabilidad del local fueron ligeramente superiores en el grupo PMC; sin embargo, tales diferencias no tuvieron significación estadística (cuadro 2).

En relación a la percepción sobre el tiempo de espera para la atención, se encontró que poco más de la mitad de las usuarias del grupo PMC $(59,1 \%)$ expresaron que dicho tiempo fue largo o muy largo; este valor fue similar al encontrado en el grupo sin PMC $(60,0 \%)$. El tiempo de espera fue percibido como aceptable por $12,5 \%$ de las gestantes que acudieron a los hospitales con PMC y por $15,1 \%$ de las gestantes que fueron atendidas en los centros sin PMC.

\section{Razones de insatisfacción}

Se estudiaron las razones por las que algunas usuarias expresaron insatisfacción (categorías muy mala, mala y regular) con la atención que recibieron en el centro. Considerando el total de las entrevistadas, la proporción fue del $32,4 \%(n=62)$ en el grupo con PMC y de $44,8 \%(n=83)$ en el grupo sin PMC. En de general, las razones de insatisfacción no difieren mucho entre los grupos de estudio, excepto en cuanto al tiempo de espera y el trato del personal (cuadro 3). La percepción del tiempo de espera como largo o muy largo constituye la razón principal de insatisfacción $(40,3 \%$ en el grupo con PMC frente al $49,4 \%$ del grupo sin PMC) seguida del inadecuado trato del personal $(22,6 \%$ en el grupo con PMC frente al 39,8\% en el grupo sin PMC).

\section{Factores asociados a la satisfacción}

El cuadro 4 muestra los factores asociados a la SU. Se aprecia que el trato del personal (razón de posibilidades $[R P]=7,25$; IC95\%: 4,07\%-12,91\%), la información que la usuaria recibe durante el control prenatal $(\mathrm{RP}=5,03$; IC95\%: 2,28\%-11,10\%) y la satisfacción de las expectativas ( $\mathrm{RP}=3,41$; IC95\%: $1,68 \%-6,92 \%$ ) son los factores que muestran mayor fuerza de asociación. La regresión logística también muestra que la implementación del PMC tiene una asociación directa, aunque de menor magnitud, con la SU. Por último, las diferencias entre los dos grupos en cuanto a la percepción de las 
CUADRO 2. Satisfacción de usuarias con la atención prenatal según grupos de estudio $(n=376)$. Evaluación de un programa de calidad en hospitales del Perú, 2000

\begin{tabular}{|c|c|c|c|c|c|c|c|}
\hline Variables del estudio & Grupo & \multicolumn{6}{|c|}{ Nivel de satisfacción (\%) } \\
\hline Satisfacción de usuaria con la atención & PMC & 0 & 0,5 & 31,9 & 58,6 & 8,9 & \\
\hline \multirow[t]{2}{*}{ Información en la consulta prenatal } & PMC & 1,0 & 0,5 & 13,6 & 62,3 & 22,5 & \\
\hline & Sin PMC & 0 & 0 & 20,5 & 64,3 & 15,1 & 0,048 \\
\hline Trato del personal & PMC & 0 & 0,5 & 30,4 & 64,9 & 4,2 & \\
\hline Limpieza del establecimiento & Sin PMC & 0 & 1,1 & 22,2 & 71,9 & 4,9 & 0,922 \\
\hline \multirow[t]{2}{*}{ Equipos del establecimiento } & PMC & 0 & 2,2 & 38,4 & 57,3 & 2,2 & \\
\hline & Sin PMC & 0,6 & 4,0 & 40,7 & 49,2 & 5,6 & 0,534 \\
\hline \multirow[t]{2}{*}{ Comodidad del establecimiento } & PMC & 0 & 4,2 & 33,5 & 57,6 & 4,7 & \\
\hline & Sin PMC & 2,2 & 3,2 & 42,7 & 47,6 & 4,3 & 0,054 \\
\hline
\end{tabular}

PMC = Programa de mejora continua

${ }^{\text {a }}$ Calculado con la prueba de suma de intervalos $U$ de Mann-Whitney. Los valores $P$ menores de 0,05 se consideran significativos.

usuarias sobre la limpieza del establecimiento, el equipamiento del hospital y el tiempo de espera para la atención prenatal no tuvieron, en este estudio, significación estadística.

\section{DISCUSIÓN}

La aceptabilidad de los servicios de control prenatal se refleja en un mayor grado de satisfacción, mayor continuidad y mayor adherencia de la gestante para cumplir las visitas programadas y las indicaciones del profesional de atención prenatal. Esto es de impor- tancia, puesto que la atención prenatal de calidad se encuentra asociada a una mayor probabilidad de parto en un centro de la red sanitaria, con reducción del riesgo de complicaciones y muertes neonatal y materna (25). Se hace necesaria la construcción de modelos explicativos sobre la SU, a fin de identificar aspectos que pueden ser modificados para mejorar la calidad de los servicios y robustecer su aceptabilidad social (26). El presente estudio pretende apoyar estas medidas.

Un estudio realizado en los servicios de maternidad hospitalarios de Finlandia encontró niveles de satisfacción de

CUADRO 3. Principales razones de insatisfacción de las usuarias de los servicios prenatales según grupos de estudio $(n=145)$. Evaluación de un programa de calidad en hospitales del Perú, 2000

\begin{tabular}{lccc}
\hline \multicolumn{1}{c}{ Razones de insatisfacción } & $\begin{array}{c}\text { Grupo PMC } \\
(n=62)\end{array}$ & $\begin{array}{c}\text { Grupo No PMC } \\
(n=83)\end{array}$ & Valor $P$ \\
\hline Tiempo de espera prolongado & 40,3 & 49,4 & 0,01 \\
Trato inadecuado & 22,6 & 39,8 & 0,001 \\
Problema de salud no resuelto & 3,2 & 2,4 & 0,41 \\
Costos elevados & 1,6 & 2,4 & 0,22 \\
Desconfianza en los médicos & 3,2 & 1,2 & 0,05 \\
Desconfianza en las enfermeras & 1,6 & 0,5 & 0,89 \\
Equipamiento inadecuado & 1,6 & 0,8 & 0,98 \\
Establecimiento distante & 0,3 & 1,2 & 0,19 \\
Otros motivos & 16,1 & 22,9 & 0,88 \\
\hline PMC = Programa de mejora continua. & & \\
a Calculado con la prueba de suma de intervalos $U$ & de Mann-Whitney. Los valores $P$ menores de 0,05 se consideran \\
significativos. &
\end{tabular}

control prenatal del $66 \%$ (27). Hall y Dornan, a través de un metaanálisis, comunicaron que el nivel promedio de satisfacción en poco más de 200 estudios, en su mayoría descriptivos realizados en consultorios clínicos ambulatorios, fue del $76 \%$ (28). Al parecer la respuesta de la gestante durante la consulta prenatal tendría particularidades que se reflejarían - aparentementeen menores niveles de satisfacción en comparación con la población en general. Este resultado ameritaría investigación, aun cuando es probable que los diversos métodos e instrumentos de medición tengan alguna influencia. Aun así, en el grupo con PMC los niveles de SU fueron mayores que en el grupo sin intervención, lo cual reflejaría que los principios y contenidos estratégicos del PMC orientados hacia una relación de cordialidad, buen trato y respeto entre el proveedor y el usuario, la mayor comunicación e información entre personas y la mejor accesibilidad administrativa para el usuario están logrando un mayor grado de aceptabilidad y satisfacción.

Las políticas públicas sanitarias del Perú procuran incrementar el uso de los servicios por la población, dando prioridad a la promoción e incremento del control prenatal y parto hospitalario de calidad, con el propósito de reducir la mortalidad materna (29). En esto contexto, minimizar los niveles de 
CUADRO 4. Factores asociados a satisfacción de usuarias de los servicios prenatales $(n=$ 376). Evaluación de un programa de calidad en hospitales del Perú, 2000

\begin{tabular}{lccc}
\hline${\text { Factores asociados a satisfacción de } \text { usuarias }^{\mathrm{a}}}^{{ }^{2}}$ & $\mathrm{RP}$ ajustada & $\mathrm{IC95 \%}$ & Valor $P$ \\
\hline Trato del personal & 7,25 & $4,07-12,91$ & 0,000 \\
Información durante la consulta prenatal & 5,03 & $2,28-11,01$ & 0,000 \\
Satisfacción de las expectativas & 3,41 & $1,68-6,92$ & 0,001 \\
Pertenecer al grupo de intervención & 2,51 & $2,1-7,3$ & 0,009 \\
Comodidad del establecimiento & 2,14 & $1,17-3,91$ & 0,013 \\
Limpieza del establecimiento & 1,83 & $0,91-3,69$ & 0,094 \\
Equipamiento & 1,30 & $0,71-2,35$ & 0,395 \\
Tiempo de espera percibido para la atención & 1,08 & $0,60-1,93$ & 0,799 \\
\hline
\end{tabular}

RP = Razón de posibilidades; IC95\% = Intervalo de confianza del 95\%.

a Ajustado según las variables edad, nivel económico, y nivel educativo. Los valores fueron estimados con análisis de regresión logística múltiple. Nivel de significación: $P<0,05$.

insatisfacción de las usuarias es importante para conseguir la aceptabilidad. Nuestro estudio evidencia que los principales motivos de insatisfacción con el control prenatal fueron el tiempo de espera prolongado y el trato inadecuado, situaciones menos frecuentes en los establecimientos que usaron el PMC. Kojo-Austin y colaboradores (27) estudiaron a gestantes finlandesas con control prenatal hospitalario, y encontraron razones similares de insatisfacción además de escasa comunicación entre el proveedor y la usuaria. Situación similar se encontró en un estudio realizado en España en la población en general (30). Esfuerzos por conseguir el buen trato, respeto, empatía y trato más humanizado en la atención a la gestante deberían formar una parte fundamental de los planes de mejora de los servicios de salud.

Identificar los aspectos de la atención prenatal que influyen en la satisfacción ayudaría a mejorar la gestión y organización de los servicios; el presente estudio muestra que el trato del personal, la información durante la consulta y la satisfacción de las expectativas - todo ello relacionado con la calidez y humanización de la atención - son valorados muy positivamente y aceptados por las usuarias. La información sobre la salud tiene una demanda cada vez mayor, encontrándose una relación directa entre el grado de disponibilidad de la información (sobre el diagnóstico, los cuidados, la conducta terapéutica y educación sanitaria) y la satisfacción $(31,32)$. Por ello, proveer información de calidad a los usuarios debería ser una conducta incorporada progresivamente a las políticas institucionales de los servicios de salud.

Otros estudios también han encontrado una asociación entre la SU y las expectativas que son satisfechas (33$35)$, y entre la SU y el trato personal $(36,37)$. Las expectativas no satisfechas disminuyen la satisfacción, no solo en el momento inmediatamente después de la consulta sino también en las consultas posteriores (32). Los servicios de salud, producto de la globalización, la complejización tecnológica, los avances de los medios de comunicación y la urbanización creciente, deben afrontar el desafío de satisfacer a una población cada vez más multicultural, con mayor grado de información y más exigente a la hora de buscar la resolución de los problemas sociales y de salud. Este reto hace impostergable la tarea de explorar las expectativas y demandas sanitarias de los usuarios a fin de traducirlas en características de la oferta.

El presente estudio tiene algunas limitaciones. Para valorar la SU nuestro instrumento ha tenido en cuenta la respuesta a una sola pregunta. Avis y colaboradores consideran que reducir la satisfacción a una sola categoría es una práctica cuestionable; no obstante, otros autores consideran que las encuestas a través de cuestionarios estructurados y preguntas de síntesis son mejores para medir la satisfacción y sus factores asociados (2). En este estudio, la satisfacción se midió junto con la percepción de las usuarias sobre otros aspectos de su itinerario de atención, con el propósito de identificar cuáles son de relevancia en su satisfacción global. La escala de Likert, utilizada en este estudio, ha mostrado mayor validez que otros métodos en cuanto a la medición del nivel de la SU. Por otro lado, la encuesta se realizó en el momento de salida de la consulta prenatal, lo cual podría generar una mayor valoración de la satisfacción debido al compromiso de la usuaria por no expresar su falta de satisfacción ("sesgo de cortesía"), situación descrita en la literatura (38). Esta situación ocurre sobre todo en los usuarios de bajo nivel educativo y pocos conocimientos sobre el centro que visitan (nuevas usuarias, por ejemplo). Sin embargo, los grupos del estudio presente son similares en cuanto a las características socioeconómicas; es más, ambos estuvieron expuestos al mismo sesgo de medición, situación que habla a favor de la validez de los resultados obtenidos. Algo similar ocurre con la exploración de las expectativas, pudiendo existir limitaciones en la medición, tal como señalan Kravitz y colaboradores (39). Estos investigadores encontraron que tiene mayor validez medir las expectativas a través de cuestionarios estructurados de antemano que por entrevistas abiertas. En el estudio presente, la prueba piloto permitió sondear las expectativas de las gestantes, lo que sirvió de base para confeccionar una lista de expectativas incluidas en un cuestionario semiestructurado.

El estado de salud de las usuarias previo a la consulta es un aspecto que nuestro estudio no ha medido. En la población de mujeres no gestantes se ha informado de una asociación entre el estado de salud previo a la consulta y la SU (40). Aun cuando la gran mayoría de nuestras entrevistadas acudió a un control prenatal de rutina (no por complicaciones), el efecto de este factor en la población objeto de este estudio se desconoce. Por lo tanto existieron limitaciones en la medición de algunos aspectos contextuales que pudieran influir en la SU. Esta situación podría generar dudas en cuanto a las diferencias encontradas, ya que estas podrían obe- 
decer a otros factores distintos a la intervención del PMC. Sin embargo, durante el período de intervención no se han documentado otras intervenciones sistemáticas y sostenidas en los hospitales estudiados. Queda como desafío establecer el papel de otros aspectos contextuales (sociales, geográficos, de población, u económicos, por citar algunos) en la SU y la aceptabilidad de la atención sanitaria.

En síntesis, el presente estudio refleja la relación entre un programa de mejora de la calidad de la atención en servicios maternos y perinatales y su efecto posi- tivo en la SU. Asimismo, ratifica la necesidad de proveer servicios de salud humanizados, flexibles y en sintonía con las demandas de una sociedad civil cada vez mejor informada. Lograr una mayor aceptación de la atención prenatal contribuiría a mejorar la salud de las gestantes y los recién nacidos.

Agradecimientos. Los autores desean agradecer el aporte financiero del gobierno del Perú y de USAID para la implementación del Proyecto 2000 y para la realización del estudio de evaluación. También desean reconocer los valiosos aportes de Francisco de la Cruz en el proceso de construcción de la base de datos y análisis básico de datos, y de Joaquín Novara en el soporte técnico del trabajo de campo y en el análisis estadístico. También agradecemos a Gaby Caro (OPS Perú) su revisión de las referencias bibliográficas, y a Luciana Chagas (OPS Brasil) la revisión global del manuscrito. Por último, nuestro agradecimiento a todo el personal de salud de los hospitales estudiados, quienes con su esmerada y continua labor hicieron del Proyecto 2000 una realidad.

\section{REFERENCIAS}

1. Caminal J. La medida de la satisfacción: un instrumento de participación de la población en la mejora continua de la calidad de los servicios sanitarios. Rev Calidad Asistencial. 2001;16:276-9.

2. Avis $\mathrm{M}$, Bond $\mathrm{M}$, Arthur A. Questioning patient satisfaction: an empirical investigation in two outpatient clinics. Soc Sci Med. 1997; 44(1):85-92.

3. Delgado A, Lopez LA, Luna JD. Influencia del modelo organizativo en la satisfacción de los usuarios. Aten Primaria. 1995;16:314-21.

4. Mira J, Aranaz J. La satisfacción del paciente como una medida del resultado de la atención sanitaria. Med Clin (Barc). 2000;114 (Supl 3): 26-33.

5. Bernhart M, Wiadnyana I, Wihardjo H, Pohan I. Patient satisfaction in developing countries. Soc Sci Med. 1999;48:989-96.

6. Carr-Hill R. The measurement of patient satisfaction. J Public Health Med. 1992;14(3):236-49.

7. Williams B. Patient satisfaction: a valid concept? Soc Sci Med. 1994;38(4):509-16.

8. Grogan S, Conner M, Norman P, Willits D, Porter I. Validation of a questionnaire measuring patient satisfaction with general practitioner services. Qual Health Care. 2000;9: 210-5.

9. Duff L, Lamping D, Ahmed L. Evaluating satisfaction with maternity care in women from minority ethnic communities: development and validation of a Sylheti questionnaire. Int J Qual Health Care. 2001;13 (3):216-30.

10. Steine S, Finset A, Laerum E. A new, brief questionnaire (PEQ) developed in primary health care for measuring patients' experience interaction, emotion and consultation outcome. Fam Pract. 2001;18 (4):410-8.

11. Lafond A. Improving the quality of investment in health: lessons on sustainability. Health Policy Plan. 1995;10:63-76.

12. Hjortdahl P, Laerum E. Continuity of care in general practice: effect on patient satisfaction. Br Med J. 1992;1287-90.
13. Hall J, Dornan M. Patient sociodemographic characteristics as predictors of satisfaction with medical care: a meta-analysis. Soc Sci Med. 1990;30(7):811-8.

14. Donabedian A. The definition of quality and approaches to its assessment. Ann Arbor, MI: Health Administration Press; 1980.

15. Sitzia J, Wood N. Patient satisfaction: a review of issues and concepts. Soc Sci Med. 1997; 45(12):1829-43.

16. Wensing M, Grol R. What can patients do to improve heath care? Health Expect. 1998;1(1): 37-51.

17. Organización Panamericana de la Salud. Reunión Regional sobre Programas de Garantía de la Calidad de los Servicios de Salud en el Marco de las Reformas Sectoriales. Programa Organización y gestión de Sistemas y Servicios de Salud. Buenos Aires, Argentina: 1998. Pp. 6-12.

18. Perú, Ministerio de Salud. Sistema de Gestión de la Calidad en Salud. Lima, Perú; 2002.

19. Perú, Ministerio de Salud. Proyecto 2000, un proyecto de innovaciones en salud: Reporte Técnico Final del equipo de Asistencia Técnica, Febrero 1995-Octubre 2002. Lima, Perú; 2003.

20. Seclen-Palacin J, Benavides B, Jacoby E, Novara J, Velásquez A, Watanabe E, et al. Efectos de un programa de mejoramiento continuo de la calidad en servicios materno perinatales en el Perú: la experiencia del Proyecto 2000. Rev Bras Saude Mater Infant. 2003;3(4):421-38.

21. Perú, Instituto Cuánto, Banco Mundial. Encuesta Nacional de Niveles de Vida. Lima, Perú: Instituto Cuánto; 2000.

22. Perú, Encuesta Demográfica y de Salud Familiar. Instituto Nacional de Estadística e Informática del Perú. Lima, Perú; 1996.

23. Velásquez A, Benavides B. Estudio comparativo de la calidad y uso de servicios de salud materno perinatales: 1997-2002. Informe Final de Evaluación. Proyecto 2000; Lima, Perú: Equipo de Asistencia Técnica; 2003.
24. Cohen J. Statistical Power analysis for the Behavioral Sciences, 2nd ed. Hillsdale, New Jersey: Lawrence Erlbaum; 198.

25. Carroli G, Rooney C, Villar J. How effective is antenatal care in preventing maternal mortality and serious morbidity? An overview of the evidence. Paediatr Perinat Epidemiol. 2001, 15 (Suppl 1):1-42.

26. Doll R. Prospects for prevention. Br Med J. 1983;285:445-53.

27. Kojo-Austin H, Malin M, Hemminki $\mathrm{H}$. Women's satisfaction with maternity health care services in Finland. Soc Sci Med. 1993; (37):633-8.

28. Hall J, Dornan M. What patients like about their medical care and low often they are asked: A meta-analysis of the satisfaction literature. Soc Sci Med. 1988;27:935-9.

29. Perú, Ministerio de Salud. Lineamientos de Políticas del Sector Salud 2002-2012. Lima, Perú; 2002.

30. Ballesteros A, García A, Fontcuberta J, Sánchez F, Pérez-Crespo C, Alcázar F. La demora en la consulta de atención primaria: se puede mejorar? Aten Prim. 2003;31(6):377-81.

31. Mendoza J, Piechulek H, Al-Sabir A. Client satisfaction and quality of health care in rural Bangladesh. Bull WHO 2001;79(6):512-7.

32. Jackson JL, Chamberlein J, Kroenke K. Predictors of patient's satisfaction. Soc Sci Med. 2001;52:609-20.

33. Baron-epel O, Dushenat M, Friedman N. Evaluation of the consumer model: relationship between patients' expectations, perceptions and satisfaction with care. Int J Qual Health Care. 2001;13:317-23.

34. Peck M, Asch D, Goold S, Roter DL, Ubel PA, McIntyre LM, et al. Measuring patient expectations: Does the instrument affect satisfaction or expectations? Med Care. 2001;39(1): $100-8$.

35. McKinley RK, Stevenson K, Adams S, Mankuscott TK. Meeting patient expectations of care: the major determinant of satisfaction with 
out-of-hours primary medical care. Fam Pract. 2002;19(4):333-8.

36. Lewis JR. Patients views on quality care in general practice: literature review. Soc Sci Med. 1994;39(5):655-70.

37. Wensing M, Grol R, Smits A. Quality judgements by patients on general practice care: a literature analysis. Soc Sci Med. 1994;38(1): 45-53.
38. Ovretveit J. Evaluating Health Intervention. Open University Press. Buckingham, Philadelphia: Blackwell Scientific; 1998.

39. Kravitz R, Callahan E, Azari R, Antonius D, Lewis CE. Asessing patients' expectations in ambulatory medical practice. J Gen Intern Med. 1997;12:67-72.

40. Da Costa D, Clarke A, Dobkin P, Senecal JL, Fortín P, Danoff D, et al. The relationship be- tween health status, social support and satisfaction with medical care among patients with systemic lupus erythematosus. Int J Qual Health Care. 1999;11 (3):201-7.

Manuscrito recibido el 18 de noviembre de 2003. Aceptado para publicación, tras revisión, el 28 de julio de 2004.
ABSTRACT

Is there a link between continuous quality improvement programs and health service users' satisfaction with prenatal care? An experience in Peruvian hospitals
Objectives. 1. To compare the level of health service user satisfaction (US) with antenatal care in hospitals where a program of continuous quality improvement (CQI) was implemented, in comparison to a reference group of patients seen at hospitals that did not participate in the program. 2. To compare the reasons for dissatisfaction in both groups of users. 3. To identify the factors associated with US.

Methods. A quasi-experimental study of a representative sample of pregnant women attending prenatal care services. The women in the intervention group $(n=$ $191)$ and the reference group $(n=185)$ were interviewed on leaving the prenatal care clinic. The dependent variable was satisfaction with prenatal care, and the independent variables were satisfaction of expectations, amiability, level of health information, perception of the equipment, waiting time, cleanliness and comfort. Descriptive and multivariate statistics were calculated.

Results. The intervention group showed higher rates of US $(67.5 \%$ versus $55.1 \%$ in the reference group, $P=0,014)$. The main reasons for dissatisfaction were long waiting times and discourteous treatment, which were more frequent in the reference group. The multivariate analysis identified cordiality of the health professionals, information provided during the visit, satisfaction of expectations and implementation of the CQI program as the factors that were significantly associated with US.

Conclusions. Our findings show that the CQI program in maternal and perinatal health services was positively associated with US. These results reinforce the need for cordiality during prenatal care contacts with providers, and the need to provide appropriate information to the user, in order to help increase acceptability of and adhesion to health care recommendations among pregnant women.

FE DE ERRATUM

Torres C y Mújica OJ. Salud, equidad

y los Objetivos de Desarrollo del Milenio. (Rev Panam Salud Publica/

Pan Am J Public Health 2004:15(6):430-439).

La redacción llama la atención de los lectores a un error en la versión publicada del artículo señalado.

P. 435, figura 3: Están invertidas las dos leyendas dentro del cuadro. El área con sombreado claro representa el "alcance completo de la meta" y el área con sombreado oscuro, la "tendencia demográfica actual”, y no a la inversa. 